\title{
Discours
}

Revue de linguistique, psycholinguistique et informatique. A journal of linguistics, psycholinguistics and computational linguistics

$17 \mid 2015$

Varia

\section{Toujours est-il que (p) ou le retour à un topique antérieur}

\section{Véronique Lenepveu}

\section{OpenEdition \\ Journals}

Édition électronique

URL : http://journals.openedition.org/discours/9071

DOI : 10.4000/discours. 9071

ISSN : 1963-1723

Éditeur :

Laboratoire LATTICE, Presses universitaires de Caen

\section{Référence électronique}

Véronique Lenepveu, «Toujours est-il que (p) ou le retour à un topique antérieur », Discours [En ligne] 17 | 2015, mis en ligne le 22 décembre 2015, consulté le 30 avril 2019. URL : http:// journals.openedition.org/discours/9071 ; DOI : 10.4000/discours.9071

\section{(c) (i) (9)}

Discours est mis à disposition selon les termes de la licence Creative Commons Attribution - Pas d'Utilisation Commerciale - Pas de Modification 4.0 International. 

Revue de linguistique, psycholinguistique et informatique

\section{Toujours est-il que (p) ou le retour à un topique antérieur}

Véronique Lenepveu

CRISCO EA 4255

Université de Caen Normandie

Véronique Lenepveu, «Toujours est-il que (p) ou le retour à un topique antérieur», Discours [En ligne], 17 | 2015, mis en ligne le 22 décembre 2015.

URL: http://discours.revues.org/9071

Titre du numéro: Varia

Coordination: Anne Le Draoulec et Josette Rebeyrolle 



\title{
Toujours est-il que (p) ou le retour à un topique antérieur
}

\author{
Véronique Lenepveu \\ CRISCO EA 4255 \\ Université de Caen Normandie
}

\begin{abstract}
On se propose d'étudier le fonctionnement sémantico-pragmatique de la locution adverbiale toujours est-il que et son rôle dans la répartition des informations et l'organisation du discours. Il apparaît que cette locution marque fondamentalement un retour à un topique de discours (Van Dijk, 1976; Charolles, 2003) après ce qu'on peut appeler « une séquence intercalée» comportant généralement l'expression d'une incertitude, ou d'une ignorance, mais pouvant également correspondre à une simple digression. Nous montrons alors comment le fonctionnement pragmatique de toujours est-il que prend son origine dans sa structure syntactico-sémantique, et s'explique notamment à partir de la valeur de persistance de toujours transposée sur l'axe de dicto (Martin, 1987). Pris dans la locution, l'adverbe d'aspect ne porte plus sur le contenu mais sur la prise en charge de l'assertion pour signaler le maintien et la réaffirmation d'un point de vue (Nølke, 1994): le point de vue persiste en dépit de ce qui vient d'être dit, et des doutes qui ont pu éventuellement être formulés entre-temps.
\end{abstract}

Mots clés: locution adverbiale, aspect de dicto, topique de discours, prise en charge, structure informationnelle

In this paper, we propose a semantic and pragmatic analysis of the French lexicalized expression toujours est-il que which marks a return to the main discourse topic (Van Dijk, 1976; Charolles, 2003). At first, through the analysis of real examples and of their typical context, it is shown that this lexicalized expression expresses a de dicto aspectual value (Martin, 1987). Then, we show it is necessary to take into account the de dicto value of this locution in order to study the information structure of discursive patterns in which it is employed. In this locution, the aspectual adverb toujours does not concern the content anymore, but the speaker's commitment. It indicates that the initial point of view (Nølke, 1994) is still valid (persistence value), in spite of what has been said meanwhile.

Keywords: french locution, de dicto aspectual value, discursive topic, discourse pattern, information structure, speaker's commitment

\section{Introduction ${ }^{1}$}

L'objet de cet article est d'étudier le fonctionnement sémantico-pragmatique de la locution adverbiale toujours est-il que en relation avec la structuration de l'information dans les textes. La notion de topique, qui nous permettra d'unifier les différents emplois de toujours est-il que, est ici appliquée au discours ${ }^{2}$ : elle désigne ce dont il est

1. Nous remercions les deux relecteurs anonymes pour leurs commentaires et suggestions.

2. Sur la notion de topique de discours, voir, entre autres, Van Dijk (1976), Marandin (1988), Van Kuppevelt (1995), Jacobs (200I), Charolles (2003), Craige (2010). 
question dans le discours pour «rendre compte du fait que les échanges parlés aussi bien que les textes développent un propos dont on s'attend à ce qu'il soit suivi et ne saute pas, sans ménagements, du coq à lâne» (M. Charolles $\left.{ }^{3}\right)$. Nous posons alors que la locution toujours est-il que marque fondamentalement un changement de topique discursif par rapport au contexte gauche immédiat, et indique simultanément un retour à un topique antérieur, après ce qu'on peut appeler «une séquence intercalée», comportant généralement l'expression d'une incertitude, ou d'une ignorance, mais pouvant également correspondre à une simple digression. Toujours est-il que clôt cette séquence intercalée, pour revenir au topique initial, et réaffirmer une proposition comme certaine, assurée, ce que nous illustrons au moyen de l'exemple ci-dessous ${ }^{4}$ :

[I] «Eh bien! comment va Mlle Nickleby? dit lord Frédérick; j'espère qu'elle est en bonne santé.

- Très bien, je vous remercie, milord, répondit Mme Nickleby commençant à se remettre, très bien. Elle $\mathrm{a}$ été assez mal pendant quelques jours après le dîner chez son oncle, et j'ai dans l'idée qu'elle aura attrapé un rhume dans ce fiacre, en revenant à la maison. Les fiacres, milord, sont si désagréables, qu'il vaut encore mieux, je crois, aller à pied. Par exemple, je me suis laissé dire qu'on peut faire condamner un cocher de fiacre à la transportation perpétuelle, quand il a un carreau cassé; eh bien! ils sont si négligents, qu'ils ont presque tous des carreaux cassés. J'ai eu une fois la figure enflée par une fluxion pendant six semaines, milord, pour avoir été en fiacre. Je crois bien que c'était un fiacre, ajouta-t-elle en réfléchissant; cependant, je n'en suis pas sûre: ce pourrait bien être un petit coupé. Tout ce que je sais, c'est qu'il était vert-bouteille, avec un grand numéro qui commençait par un zéro et finissait par un neuf. Non, au contraire, il commençait par un neuf et finissait par un zéro; bien, c'est cela! Et par conséquent, on pourrait tout de suite savoir au timbre, pour peu qu'on voulût s'en informer, si c'était un fiacre ou un petit coupé. Enfin, toujours est-il qu'il y avait un carreau cassé, et que j'en ai eu la figure enflée pendant six semaines. [...]»

(Ebooks libres et gratuits: Charles Dickens, Vie et aventures de Nicolas Nickleby [1838I839], trad. fr. P. Lorain, Paris, Hachette, I845, t I, chap. XXVI)

Une structure discursive se dégage ainsi reliant un point de vue initial et la réaffirmation de ce point de vue (la notion de point de vue étant prise ici au sens de Nølke, 1994) ${ }^{5}$ :

3. Voir la présentation du projet Labex «TransferS» dirigé par M. Charolles (UMR LATTICE) sur les marqueurs de changement de topique, consultable à l'adresse suivante: http://transfers.ens.fr/articleI55. html. Ma recherche s'inscrivant dans le cadre de ce projet, je remercie vivement tous les participants pour leurs commentaires et suggestions, les erreurs qui subsistent sont bien sûr de mon fait. Je remercie également, pour leurs suggestions, les collègues et doctorants présents à la deuxième rencontre interuniversitaire Caen-Neuchâtel (2I mai 20I5).

4. Le contenu asserté et réaffirmé figure en gras dans les exemples attestés proposés.

5. Dans la perspective polyphonique de Nølke (I994), un point de vue comprend un contenu propositionnel et un jugement portant sur ce contenu, le jugement consistant en la validation de la proposition. 
- point de vue initial;

- séquence intercalée = contient l'expression d'une incertitude, un aveu d'ignorance, ou une simple digression;

- toujours est-il que+ réaffirmation du point de vue initial.

Toujours est-il que ne supportant pas la négation (*toujours n'est-il pas que, voir Hansen, 2004 et 2008: 199), et que+phrase ne pouvant y jouer le rôle d'une subordonnée, on accorde à cette suite le statut de locution adverbiale ${ }^{6}$. Cette locution a fait l'objet d'études sémantiques approfondies, en particulier de la part de Nguyen (1986a), Hansen (2004 et 2008) et Buchi (2007). Les études de Hansen et Buchi aboutissent à des conclusions convergentes:

I. On retrouve la valeur sémantique de l'adverbe toujours dans la locution, mais intervenant au niveau de la prise en charge énonciative (sur l'axe de dicto, selon Martin, 1987: 120-I24).

2. La locution présente deux emplois en contexte, qui sont non exclusifs l'un de l'autre: a) un emploi "concessif» (que Buchi [2007] appelle aussi «assertif»), où la locution est synonyme de «n'empêche que», et b) un emploi «thématique», signalé par Hansen (2004: 49; 2008: 200) et considéré comme dérivé du précédent par Buchi (2007: I20), dans lequel la locution marque un «retour au thème principal après une digression» (Hansen, 2004: 49).

Ces études divergent cependant quant à la valeur de toujours dans la locution toujours est-il que, puisqu'il s'agit de la valeur temporelle de permanence selon Hansen (2004: 52), et de la valeur aspectuelle de persistance selon Buchi (2007), qui suit Martin (1987).

Nous argumenterons, dans cet article, en faveur des thèses suivantes:

I. La valeur de toujours dans la locution est bien la valeur aspectuelle de persistance (et non de permanence). Pour décrire toujours est-il que dans son fonctionnement typique, nous reprenons l'hypothèse de Martin (1987: I20-I24), selon laquelle le fonctionnement pragmatique de cette locution peut s'expliquer à partir de la valeur de persistance de toujours transposée sur l'axe de dicto. En d'autres termes, l'adverbe toujours pris dans la locution, ne porte plus sur le contenu mais sur la prise en charge de l'assertion: le point de vue «persiste» en dépit des doutes ou des interrogations qui ont pu être formulés entre-temps.

2. La locution marque dans tous les cas (et pas seulement dans son emploi thématique) le retour à un topique antérieur, par-delà une séquence intercalée. C’est uniquement quand les énoncés contenus dans cette séquence

6. Sur le figement des locutions prédicatives, voir Nøjgaard (I992: 132-I33), et sur la postposition du sujet dans un énoncé comportant un adverbial en position initiale, voir Guimier (1996: 43-95). 
ne sont pas présentés sous une modalité assertive catégorique (mais sous la modalité du doute ou de l'hypothèse) que se produit un effet concessif, provenant de ce que nous appellerons une «discordance modale». En l'absence d'une telle discordance, la locution marque simplement la fin de ce qui apparaît comme une digression.

3. Dans les cas de discordance modale, la locution n'indique pas seulement le retour au topique antérieur, mais la réaffirmation d'un point de vue déjà communiqué. Cette dernière hypothèse ne peut être validée que sur la base de l'étude des contextes gauche de la locution, parfois très étendus. Une attention toute particulière sera portée aux formes diverses selon lesquelles ce point de vue initial est communiqué (car il n'est pas toujours explicitement asserté), et réaffirmé.

La structure de l'article est la suivante: nous précisons l'hypothèse d'une portée de dicto de la valeur aspectuelle de persistance marquée par toujours au sein de la locution (partie 2). Une étude de l'évolution de la formation de cette locution relativement récente, à partir de Frantext intégral ${ }^{7}$, et sur la base des travaux de Buchi (2007), confirmera cette hypothèse (partie 3). Puis, nous examinerons la forme syntaxique que peut prendre le segment de texte qui précède immédiatement la locution (questions, hypothèses, verbes de doute...) pour nous intéresser ensuite à la relation entre le point de vue initial et le point de vue réaffirmé, ceci afin d'expliquer le rôle spécifique de toujours est-il que dans la répartition des informations et l'organisation du discours (partie 4).

\section{Toujours est-il que (p) ou un point de vue qui persiste}

\subsection{Quelles conditions d'emploi pour toujours est-il que?}

Souvent répertoriée chez les linguistes en tant que locution adverbiale concessive ${ }^{8}$ (Morel, I996: I26; Nøjgaard, I992: 484-485) ${ }^{9}$, la locution toujours est-il que a été décrite par le grammairien R. Le Bidois comme signalant ce que nous appellerons, pour notre part, une discordance «modale» entre propositions:

Toujours est-il (que) sert à introduire un fait ou un jugement que l'on pose comme certain, en vive opposition avec d'autres faits qui viennent d'être présentés sous le signe de l'hésitation, de l'incertitude ou de la probabilité. (Le Bidois, 1952: I04)

7. ATILF. Base textuelle Frantext. En ligne à l'adresse suivante: http://www.frantext.fr/.

8. Dans les classifications de Nøjgaard (1992) et de Morel (1996), la locution toujours est-il que est rapprochée du marqueur de concession à choix aléatoire quoi qu'il en soit, c'est-à-dire d'une locution utilisée, nous citons Morel (I996: I26), "pour marquer qu'on ne veut pas discuter du bien-fondé de ce qui vient d'être dit».

9. Si cette locution n'a jamais fait l'objet d'une étude isolée, elle est en revanche très souvent analysée dans les travaux visant à identifier les différents emplois pragmatiques de toujours (Cadiot et al., 1985; Nguyen, I986a et b; Hansen, 2004 et 2008) ou bien sa valeur de base en langue (Fuchs, 1988; Franckel, I989; Nemo, 2000; Hansen, 2004 et 2008). 
Les exemples ci-dessous illustrent bien cette discordance au niveau modal entre la proposition dans laquelle la locution s'insère et celles qui la précèdent, l'incertitude étant exprimée respectivement sous la forme d'une série d'interrogatives directes (exemple [2]), puis au moyen de l'adverbe de modalité épistémique peut-être (exemple [3]):

[2] On chercha en vain le philosophe norvégien. Une colique l'avait-elle saisi? Avait-il eu peur de manquer le train? Un aéroplane était-il venu le chercher? Avait-il été emporté dans une Assomption? Toujours est-il qu'il avait disparu sans qu'on eût le temps de s'en apercevoir, comme un Dieu.

(Marcel Proust, Sodome et Gomorrbe, t. II, chap. II, in À la recherche du temps perdu, t. V, Paris, Gallimard, 1922; cité par Le Bidois, 1952: 105)

[3] C'est peut-être lui qui m'a rendu si malade. Enfin, toujours est-il que le lendemain de ce repas, je ne pouvais plus me lever.

(Frantext: Louis-Ferdinand Céline, Voyage au bout de la nuit [1932], Paris, Gallimard, 2000, p. 172)

Plus récemment, Hansen (2004: 49-50) a identifié deux emplois pour cette locution, même si dans les deux cas de figure, le locuteur indique que le contenu de l'énoncé introduit avec toujours est-il que «demeurera en force quelles que soient les circonstances»:

- L'emploi est dit «concessif» (concession faible) quand le contexte qui précède évoque des attentes contredites par l'énoncé introduit par toujours est-il que. Soit l'exemple [4]:

[4] Il est possible que Jean réussisse brillamment à l'examen. Toujours est-il que son prof ne l'aime guère ${ }^{10}$.

(Hansen, 2004: 49)

- L'emploi est dit «thématique» quand la locution ne contredit aucune attente particulière; il sert alors à marquer le retour au thème principal, et à structurer le discours en clôturant une digression. Selon Buchi (2007), le deuxième emploi serait issu d'une intersubjectification au sens de Traugott et Dasher (2002) du premier emploi : ce qui se trouve relativisé n'est plus la valeur de ce qui vient d'être énoncé, mais «l'attention que le co-énonciateur est censé y porter» (Buchi, 2007: I2I). Soit l'exemple [5]:

[5] [...] le quartier était plutôt discrédité sur le marché des locations. Période de crise... on se demande d'ailleurs quelle période n'est pas de crise? Toujours est-il qu'aller percher dans le XIII e ça vous classait chez les loquedus.

(Alphonse Boudard, Mourir d'enfance [1995]; cité par Hansen, 2004: 49)

10. L'interprétation proposée par l'auteur est la suivante: on aurait pu s'attendre à ce que, du moins, Jean ne déplaise pas à son professeur, les professeurs ayant tendance à apprécier les étudiants potentiellement brillants. 
À la suite de Hansen, Buchi (2007: I2O-I2I) propose deux paraphrases permettant de distinguer également deux types d'emploi:

- Un emploi paraphrasable par «n'empêche que, reste que». À la différence de Hansen (2004), Buchi (2007) parle, dans ce cas, non pas d'emploi «concessif» mais d'emploi «assertif», en référence à la classification de Guimier (1996):

[6] Il se trouvera de bons esprits pour plaider la coïncidence, toujours estil que mon père s'en fut avec ma sœur le jour qu'elle se découvrit une femme.

(Frantext: Yves Berger, Le Sud, Paris, B. Grasset, 1962, p. 33)

- Un emploi paraphrasable par «pour en revenir à ce qui a été dit tout à l'heure»:

[7] - Je viens de faire une curieuse rencontre, dis-je à ma sœur, tout en lui servant un peu de rosé du Béarn. Vous rappelez-vous ce repas de noce?

- Oh oui! Vous m'aviez d'ailleurs bien rendu service!

- C'était peu de chose... Toujours est-il que j’ai rencontré le jeune homme qui avait à payer le gage, vous vous souvenez?

(René-Victor Pilhes, La rbubarbe [1965]; cité par Buchi, 2007: I2I)

Qu'elle prenne en contexte un effet de sens concessif ou non, nous avons observé que la locution toujours est-il que a systématiquement pour fonction de marquer le retour à un topique antérieur (comme il apparaîtra dans les exemples que nous allons analyser). Cette fonction peut être expliquée à partir de la valeur de persistance de toujours.

\subsection{Valeur de persistance et axe de dicto}

Rappelons tout d'abord le test de la négation permettant de lever l'ambiguïté entre les deux valeurs associables à toujours, à savoir permanence et persistance ${ }^{11}$. Soit les deux couples d'énoncés ci-dessous:

[8] Il l'a toujours aimée ( $\approx$ il l'a aimée tout le temps)/Il ne l'a pas toujours aimée (toujours de permanence)

[9] Elle l'aime toujours ( $\approx$ elle l'aime encore au moment considéré; voir Buchi, 2007: $\left.113^{12}\right) /$ Elle ne l'aime plus (toujours de persistance)

Alors que pas toujours ne peut affecter que la valeur de permanence de toujours (exemple [8]), la négation de la persistance est exprimée avec ne... plus (exemple [9]),

11. Précisons que peut se greffer en contexte un effet de sens itératif.

12. Sur les différences entre toujours et encore, voir Muller (1999: 228-229), Fuchs (1988) et Hansen (2008: I48). 
tandis que toujours pas marque la persistance d'un prédicat nié (Elle ne l'aime toujours pas); voir Martin (1987: 121) et Hansen (2008: 136, I48).

Cette idée de persistance marquée par toujours au niveau de re, c'est-à-dire au niveau des faits, peut se transposer sur l'axe de dicto, c'est-à-dire sur l'axe de la prise en charge des faits, comme l'indique Martin (1987):

Dans un exemple comme celui-ci : Moi je vais emporter les cent balles et la monnaie... Hein?... Ça fera toujours mon voyage... (Céline, Mort à crédit, 662), la vérité de ça fera mon voyage ne saurait être mise en cause; elle persiste quoiqu'il advienne. Sans préjuger des conséquences du fait que «je vais emporter les cent balles et la monnaie», une chose est sûre : «ça fera mon voyage». Cette dernière proposition, je puis la prendre en charge indéfiniment sans rupture imprévisible. (Martin, 1987: I2I)

Deux arguments syntaxiques corroborent cette analyse chez Martin (1987) et Buchi (2007). Tout d'abord, la négation lorsqu'elle est possible, se fera avec toujours pas:

[io] Prosper - Et il faut voir l'effet que ces choses-là font en province, quand on les raconte.

L'Huissier - Qui est-ce qui les raconte? Ce n'est toujours pas les députés.

(Alfred Capus, Les favorites [19II] ; cité par Martin, 1987: I2I-I22; d'après Damourette et Pichon, 1987, vol. 7: § 2974)

Ensuite, on retrouve dans les emplois pragmatiques de toujours l'incompatibilité observée entre toujours persistant et un aspect perfectif:

[II] Ça fait pas une fortune, mais c'est toujours ça [...].

Ça n'a/avait/aura pas fait une fortune, ${ }^{*}$ mais ça a/avait/aura toujours été ça.

(Buchi, 2007: IIO, II6)

La valeur de persistance l'emporterait également sur la valeur de permanence dans les tours $p$ toujours est-il que $q^{13}$ (Martin, 1987: 122), la vérité de $q$ "persistant» quelle que soit la valeur de $p$.

Hansen (2004 et 2008) rattache cependant l'emploi de toujours dans la locution toujours est-il que à sa valeur modale (observable dans «Menton, c'est toujours la France», Hansen, 2008: I80), qui dériverait elle-même de sa valeur temporelle de permanence («l'idée de validité globale véhiculée par l'adverbe s’appliquerait alors au niveau de l'assertabilité de la proposition, plutôt qu'au niveau de son contenu», Hansen, 2004: 52). Pour trancher la question, on peut recourir aux

13. L'emploi méta-énonciatif de toujours quand l'adverbe figure dans la locution toujours est-il que est également identifié chez Fuchs (I988: I46) et, dans une perspective culiolienne, ramené, comme tous les emplois dits modaux, à sa valeur de base: "parcours indéfini avec impossibilité d'assigner un seuil qui modifie la situation, qui inverse la notion et son assertion». La locution quoi qu'il en soit apparait dans la paraphrase proposée pour toujours est-il que A (A est pris comme notion): «quoi qu'il en soit, on ne peut pas dire que non- $A$, rien ne permet de modifier $A$ en non- $A »$. 
paraphrases. On admet en effet que la valeur de permanence de toujours est glosable par constamment, continuellement, tandis que la valeur de persistance est exprimable par rester, demeurer. Ainsi la phrase «Pierre est toujours malade» peut-elle être interprétée comme marquant la permanence de l'état de Pierre ou bien sa persistance. Dans le premier cas, toujours peut être remplacé par constamment, continuellement. Dans le second, «rester, demeurer malade» peuvent être substitués à «être toujours malade». Transposées sur l'axe de dicto, ces expressions vont se trouver respectivement transformées en «il est constamment, continuellement vrai que p», et «il reste, demeure vrai que p». Or, l'expression «toujours est-il que $\mathrm{p}$ » peut se trouver remplacée par «il reste, demeure que p», ou, dans l'emploi concessif, par «il n'en reste, demeure pas moins vrai que p», mais jamais par «il est constamment, continuellement vrai que p». C'est pourquoi nous concluons que c'est bien la valeur de persistance de toujours qui est à l'œuvre dans la locution toujours est-il que. Dans l'emploi typique de la locution, toujours indique, sur l'axe de dicto, la persistance de la validité d'un point de vue initial.

Cette hypothèse d'un fonctionnement de la valeur de persistance de toujours sur l'axe de dicto se confirme si l'on observe l'évolution de la formation de la locution toujours est-il que, même si celle-ci a perdu de sa compositionnalité au terme d'un processus de grammaticalisation (Hansen, 2004; Buchi, 2007).

\section{Formation de la locution toujours est-il que en diachronie à partir de Frantext}

À partir de Frantext, il est possible de dégager un schéma d'évolution diachronique en trois étapes:

- première étape: il est toujours vrai/certain/sûr... que (fin XVII jusqu'au milieu du XVIII');

- deuxième étape: toujours est-il vrai/certain/sûr... que (fin XVII ${ }^{e}$ jusqu'au milieu du XIX');

- troisième étape: toujours est-il que (depuis le milieu du XVIII).

\subsection{Première étape: il est toujours vrai/certain/sûr... que} avec une valeur de dicto

Antérieurement à l'apparition de toujours est-il que, on trouve des emplois de la tournure impersonnelle il est toujours vrai que avec une valeur de dicto à la fin du XVII et ce jusqu'au milieu du XVIII ${ }^{e}$. Précisons qu'avec une valeur de re, il est toujours vrai que porte sur le contenu, et toujours exprime la permanence, il est toujours vrai que $p$ est alors paraphrasable par «il est vrai que toujours p». Soit un exemple:

[I2] Dès lors la rationalité d'une méditation non seulement ne suffit pas à caractériser la liberté, mais ce n'est pas nécessaire. Il est toujours vrai que délibérer c'est élever nos 
motifs à la clarté et à la distinction; mais il n'est pas toujours vrai que celles-ci soient conformes à ce qu'il est convenu d'appeler la rationalité.

[Il est toujours vrai que délibérer c'est élever nos motifs à la clarté et à la distinction $\approx$ il est vrai que délibérer c'est toujours élever nos motifs à la clarté et à la distinction.]

(Frantext: Paul Ricœur, Philosopbie de la volonté, Paris, Aubier, t. I, Le volontaire et l'involontaire, 1949, p. I51)

À l'inverse, dans l'emploi de dicto, il est toujours vrai que p n'est pas paraphrasable par «il est vrai que toujours p», mais par «ce qui reste vrai, c'est que p», et la locution signale la réaffirmation d'un point de vue ${ }^{14}$ :

[13] Lettre de Mme de Sévigné à Mme de Grignan

À Rennes, dimanche 29 avril 1685

[...] Je me fusse servie des généreuses offres de Mme de Marbeuf, qui sont aussi sincères qu'elles sont solides, et je m'en servirais encore sans balancer si ma jambe, comme par malice, ne se guérissait à vue d'oil. Vous savez ce que c'est aussi que de se charger de rendre ce qu'on prend si agréablement. Ainsi je vais aux Rochers observer la contenance de cette jambe, qui est présentement sans aucune plaie ni enflure. Elle est tout amollie et, pour la figure, elle est entièrement comme sa compagne qui, depuis près de six mois, était sans pareille. La couleur n'est pas agréable; la lessive ne la blanchit pas, ni l'eau d'arquebusade. Il y a encore quelques marques de fructus belli, qui dureront longtemps, mais ce n'est que les places des feux qui sont passés. Je ne sais si c'est la sympathie des petites herbes qui me guérit à mesure qu'elles pourrissent en terre. J'avais envie d'en rire, mais les capucins en font tous les jours des expériences; je voudrais bien savoir ce qu'en dit Alliot. Je ne sais donc si c'est la cérémonie de ces petits enterrements deux fois le jour, ou si c'est la lessive ou le baume, mais il est toujours vrai que je n'ai point été comme je suis, et que si cette guérison n'est pas véritable, je n'en irai chercher qu'auprès de vous. Voilà, ma chère bonne, des vérités dont je vous conjure de ne pas douter. (Frantext: Mme de Sévigné, Correspondance, Paris, Gallimard, t. 3, I680-1696, 1978, p. $\left.195^{-196)}\right)^{15}$

Précisons qu'à cette étape, on observe des variations de l'adjectif à valeur modale (il est toujours vrai/sûr/certain/évident/constant... que), ainsi que des variations temporelles, ce qui est illustré dans les exemples ci-dessous:

14. La construction impersonnelle est soulignée.

15. Première occurrence attestée dans Frantext de il est toujours vrai que avec une valeur de dicto (I685). La dernière occurrence avec un emploi de dicto est attestée en I733. On trouve cependant un emploi de dicto polyphonique attesté au XIX ${ }^{\mathrm{e}}$. Dans l'exemple suivant, il est toujours vrai que $p$ réfère au discours de l'autre: Cher Ambroise, quand te verrai-je? Il est toujours vrai que tu viens à Paris, n'est-ce pas? (Frantext: A. Gide, P. Valéry, Correspondance (1890-1942) [1942], Paris, Gallimard, I955, p. I74, Gide à Valéry, oct. I892). 
[14] L'acteur qui récitoit et l'acteur qui faisoit les gestes, étoient donc obligez de suivre une même mesure dont l'un et l'autre devoient également observer les tems. Nous avons vû dans Quintilien qu'on tâchoit d'établir une proportion entre les gestes et les mots que disoit l'orateur, de maniere que son action ne fut ni trop fréquente ni trop interrompuë. On peut croire que cette idée venoit de ce que l'acteur qui récitoit sur le théatre, ne devoit dire qu'un certain nombre de mots, tandis que l'autre acteur chargé de la gesticulation faisoit un certain geste. Le premier devoit dire apparemment un plus grand nombre de mots lorsque le second faisoit un autre geste. Quoi qu'il en soit, il est toujours constant que l'un et l'autre suivoient les temps d'une même mesure batüe par le même homme, qui avoit sous les yeux les vers qui se récitoient, et dont les syllabes marquoient les temps, comme on l'a vû. (Frantext: Abbé Jean-Baptiste Dubos, Réflexions critiques sur la poésie et la peinture, Paris, J.-P. Mariette, 1733, p. 238-239)

[I5] Or suivant les apparences, cette cérémonie ne se sera faite qu'après que l'église dont Clovis avoit commencé la construction, eut été achevé de bâtir, et quand le mausolée où le fondateur et sa famille devoient reposer, eut été fini. Un édifice tel que celui-là n'est point l'ouvrage d'une seule année, quand même les conjonctures n'y apporteroient pas aucun retardement. D'ailleurs la vie de sainte Geneviéve dit positivement, que l'église de saint Pierre et de saint Paul, laquelle porte aujourd'hui le nom de cette sainte, fut bien commencée par Clovis, mais qu'elle ne fut achevée qu'après sa mort, et par les soins de sa veuve la reine Clotilde. Ainsi, supposé que Clovis, comme le dit l'auteur des gestes, ait fait commencer la basilique des saints apôtres, lorsqu'il partit en cinq cens sept pour aller faire la guerre aux ariens, il sera toujours vrai qu'elle n'étoit pas encore finie quand ce prince mourut en cinq cens onze.

(Frantext : Abbé Jean-Baptiste Dubos, Histoire critique de l'établissement de la monarcbie françoise dans les Gaules [1734], Paris, Nyon fils, 1742, p. 256)

[16] Il est vrai que tant que Clovis a vécu, le diocése de Langres a toujours été sous la domination des bourguignons; mais l'abbaye du Moustiers-Saint-Jean qui est bâtie à l'extrêmité septentrionale de ce diocèse, comme l'observe le pere Daniel, pouvoit bien être sur le territoire de Clovis. Quoique les bourguignons tinssent la ville capitale de la cité de Langres et la plus grande partie du plat-pays de cette cité, il pouvoit bien se faire que les francs en eussent occupé quelque canton après le désastre de Syagrius. Nous l'avons observé déja, dans des revolutions pareilles à celle qui arriva pour lors, les bornes légales des provinces et des autres districts, ne sont pas toujours respectées: elles ne sont pas toujours celles qui limitent les acquisitions des conquerans. Ils les étendent jusques aux fleuves, aux montagnes et aux autres bornes naturelles, capables par elles-mêmes d'arrêter les progrès d'un vainqueur. Quoiqu'il en ait été, il sera toujours certain que l'abbaye du Moustiers-Saint-Jean étoit du moins voisine de la frontiere des francs. Ainsi elle pouvoit très-bien tenir des terres et d'autres possessions dans les pays de l'obéissance de Clovis.

(Frantext: Abbé Jean-Baptiste Dubos, Histoire critique de l'établissement de la monarcbie françoise dans les Gaules [1734], Paris, Nyon fils, 1742, p. I08-109) 


\subsection{Deuxième étape: toujours est-il vrai/sûr/certain... que}

Fin $\mathrm{XVII}^{\mathrm{e}}$, la forme inversée entre en concurrence avec les tournures impersonnelles répertoriées ${ }^{16}$. Les deux formes (inversées/non inversées) coexistent jusqu'au milieu du XVIII ${ }^{\mathrm{e}}$ siècle, mais l'inversion du sujet paraît être un moyen de contraindre l'interprétation, car la forme inversée va marquer systématiquement la persistance au niveau de dicto, et signaler la réaffirmation d'un point de vue. C'est-à-dire que la forme inversée supprime toute possibilité d'ambiguïté, la lecture ne peut être que de dicto:

[I7] En conséquence donc du principe établi, qu'il ne sçauroit s'élever de nouveaux habitans dans un état qu'à proportion des moyens de subsistance, que plus cette subsistance est volontairement resserrée par ceux qui occupent le terrein, plus il en reste pour fournir à une nouvelle peuplade, il seroit impossible de nier que toutes autres choses mises à part, les établissemens des maisons religieuses ne soient très-utiles à la nombreuse population. Que ce soit de par le roi, de par saint Benoît ou saint Dominique, qu'un grand nombre d'individus s'engagent volontairement à ne consommer que cinq sols par jour, toujours est-il vrai que ces sortes d'institutions aident fort à la population, simplement en donnant de la marge et laissant du terrein à d'autres plançons.

(Frantext: Marquis Victor de Mirabeau, L'ami des hommes ou Traité de la population [I755], Avignon, I756, p. 6o)

[i8] Thebes le pouvoit disputer aux plus belles villes de l'univers. Ses cent portes chantées par Homere sont connuës de tout le monde. Elle n'estoit pas moins peuplée qu'elle estoit vaste, et on a dit qu'elle pouvoit faire sortir ensemble dix mille combatans par chacune de ses portes. Qu'il y ait si l'on veut de l'exageration dans ce nombre, toûjours est-il asseûré que son peuple estoit innombrable. Les grecs et les romains ont célebré sa magnificence et sa grandeur, encore qu'ils n'en eussent veû que les ruines: tant les restes en estoient augustes.

(Frantext: Jacques-Bénigne Bossuet, Discours sur l’histoire universelle, Paris, S. MabreCramoisy, I68I, p. 455)

[19] Et afin que vous n'en doutiez point, on vous fera voir par le témoignage de toute l'antiquité, que les apôtres établirent par leur prédication, une eglise à Jérusalem. II. si vous vous informez du tems: c'est dans l'espace de trois ans, que les miracles de Jesus Christ, sa mort, sa résurrection et son ascension doivent être arrivés; et c'est quelques semaines après ce dernier événement, que les apôtres commencérent de prêcher publiquement à Jérusalem.

16. Frantext ne nous permet pas d'affirmer la coexistence des formes il est toujours vrai que (avec une valeur de dicto) et toujours est-il vrai que puisque la dernière occurrence de il est toujours vrai que avec un emploi de dicto est attestée en I733, et que c'est en 1755 que nous trouvons la première occurrence avec inversion du sujet pronominal. En revanche, on peut affirmer que les formes toujours est-il certain/évident/constant/sûr que (avec ou sans accent circonflexe sur toujours) entrent en concurrence avec la forme non inversée de la fin du XVII jusqu'au milieu du XVIII ${ }^{e}$. Parmi les adjectifs à valeur modale qui peuvent figurer dans ce tour, nous avons trouvé : vrai, certain, évident, sûr, constant, assuré, clair, manifeste, incontestable et bors de doute. 
III. si vous voulez savoir quels sont ces témoins qui déposent que ces faits sont véritables: on en produit un très-grand nombre qui vivent et qui ont conversé avec Jesus Christ.

[...]

Les apôtres ne se contentent pas de prêcher toutes ces choses, ils les écrivent, et leurs ecrits sont portés en tous lieux. [...] Je veux qu'on ait composé ces livres quarante, cinquante, soixante ans après la mort de Jesus Christ; toûjours est-il évident, qu'avant ce tems il y avoit une eglise à Jérusalem, qui avoit été fondée par la prédication des apôtres $[\ldots]$.

(Frantext: Jacques Abbadie, Traité de la vérité de la religion cbrétienne, Rotterdam, R. Leers, I684, vol. 2, p. 64-66)

On retrouve les mêmes variations adjectivales (exemples [18], [19] ci-dessus) et temporelles (exemple [20] ci-dessous; Buchi [2007: 120] mentionne également la forme toujours était-il clair que [1780]).

[20] Le tribunal de Saintes a ajouté un motif subsidiaire d'après lequel c'est par le mérite même du fond qu'il aurait établi le principe d'incompétence. Mais à cet égard une seule chose était à examiner: y avait-il eu des coups portés par le mari à son épouse? L'affirmative n'était pas douteuse. Que les coups aient été plus ou moins violens, qu'ils aient eu des suites plus ou moins fâcheuses, c'est un point qui devait augmenter ou diminuer la peine; mais toujours était-il certain qu'il y avait un délit et conséquemment toujours était-il incontestable que le Ministère public avait action pour le poursuivre.

(Journal du Palais présentant la jurisprudence de la Cour de cassation et des cours d'appels de Paris et des autres départements, sur l'application de tous les codes français aux questions douteuses et difficiles, Paris, t. 2, 1825, p. 579)

Enfin, on observe la possibilité d'incises, indices d'un figement encore assez relatif:

[2I] - Que ce soit cela ou autre chose, toujours est-il certain, monsieur le conquérant, que ce soir il n'y aura pas entre nous de raccommodement dans ce sens-là.

(Frantext: Jean-Baptiste Louvet de Couvray, Une année dans la vie du chevalier de Faublas [1787], Paris, Gallimard, 1960, p. 597)

\subsection{Troisième étape: toujours est-il que}

À partir du milieu du XVIII e, l'inversion du sujet peut se trouver associée à une disparition de l'adjectif (Hansen [2008: 199] donne la même date d'apparition de la locution lexicalisée). La suite toujours est-il que entre alors en concurrence avec la forme inversée avec adjectif ${ }^{17}$ et signale aussi la réaffirmation d'un point de vue:

17. La forme inversée avec adjectif se rencontre jusqu'au milieu du XIX ${ }^{\mathrm{e}}$. Soit la dernière occurrence attestée dans Frantext pour chacune de ces formes: toujours est-il asseûré que (I68I), toûjours est-il évident que (I684), toujours est-il sûr que (1826), toujours est-il vrai que (1835), toujours est-il constant que (I843), toujours est-il certain que (1863). La suite toujours est-il hors de doute que est cependant attestée en I920. 
[22] Vous convenez qu'il faut peupler, et fortifier vos colonies; je crois qu'il en est à leur égard, comme d'un champ qu'il faut défricher, labourer, fumer et semer, avant que de rien recueillir. Si donc vous envoyez sans cesse à vos colonies sans songer à en rien retirer; si vous leur donnez des chefs d'une probité reconnue, d'une autorité naturelle et prise dans la gravité des mœurs, patients, généreux, sçachant estimer les hommes, découvrir, et cultiver leurs talens; si vous payez bien ces chefs, et les mettez à même de tenir un grand état sans percevoir aucuns droits onéreux sur le commerce, et moins encore sur la débauche et les folies des colons; si vous les y laissez long-temps avec une autorité entiére; si, fermant l'oreille aux plaintes et cabales des vauriens toujours soûtenus dans les cours, vous dèshonorez, quand ces chefs reviendront, ceux qui se seront enrichis dans leurs places, et récompensez ceux qui reparoîtront avec la pannetiére et la houlette, dormez alors sur les détails, et ne veillez qu'aux secours principaux, et au choix des dépositaires de votre autorité; vos colonies se peupleront et se renforceront d'elles-mêmes avec une rapidité, dont les progrès vous étonneront.

Mais, dira-t-on, ce systême spécieux dans l'exposition seroit dans la pratique précisément le moyen de relâcher tous les chaînons qui lient ces parties éloignées à la masse, d'écarter les rapports des provinces à la capitale, et de faire de ces plantations cultivées avec tant de soin, des états distincts et séparés de la métropole. Oh! Nous voici revenus à l'admirable axiome, divide, et impera; de crainte que les colons ne deviennent trop indépendants, il faut les maintenir foibles et grossiers, les livrer à un gouvernement intercadent, leur rendre enfin le joug habituel; c'est ainsi, dit-on, que Genes a gouverné la Corse. Mais je veux que cette façon de faire vous réussisse en ce point; toujours est-il, que vous êtes convenus de la nécessité de peupler et de fortifier les colonies. vous sentez vous-même que votre méthode actuelle n'y est pas propre [...].

(Frantext: Marquis Victor de Mirabeau, L'ami des hommes ou Traité de la population [1755], Avignon, I756, p. 366-369)

[23] À vrai dire, depuis quelque temps, mes recherches m'entraînaient fort loin des domaines de Mlle Brichs. Je pourrais préciser, rappeler que notre rencontre date de 1947, époque de la parution de mon Histoire comparée des religions du Salut, mais je devais alors montrer que j'étudiai jusqu'en 1952 environ les livres et les doctrines des Bâbys enseignées par Mirza-Aly-Mohammed, le Bâb lui-même, pour vérifier l'hypothèse d'Arthur de Gobineau selon laquelle le bâbysme aurait été nourri des idées de Spinoza, par le canal des Juifs de Schyraz. Ceci me conduirait hors de mon propos, mais suffirait à révéler la fascination que Mlle Brichs exerça sur moi durant les années 1947-1950. Car enfin, je n'avais plus maintenant aucun motif plausible de la rencontrer; elle ignorait tout de la langue vulgairement nommée «persane», je ne lui connaissais pas d'intérêt particulier pour Spinoza, et aucun des langages dont elle était maître ne touchait, de près ou de loin, à la doctrine du Bâb, hormis l'allemand, qui m'est naturellement familier.

Surtout, le peu d'années ouvertes devant moi pour mener à bien ma tâche immense m'interdit absolument toute relation inutile avec mes contemporains. Or, je remarque, 
à feuilleter mes agendas, que j'ai rencontré Mlle Brichs tous les mois, pendant près de trois ans, et qu'elle seule (je ne parle pas de ma sœur, dont les visites épisodiques et fort courtes ne me distraient en rien) a su enlever à mon travail une part non négligeable, part émouvante, part mystérieuse encore aujourd'hui dans ma mémoire, sur laquelle, jusqu'à mon dernier souffle, je ne cesserai de m'interroger. Si fallacieuse que soit la grandeur des tâches que l'Homme s'impose, - et il les sait minuscules, - il ne peut toujours saisir la raison des incidents qui l'en divertissent.

Toujours est-il, - je reviens sur le début de ce récit - que je fis sa connaissance au printemps de $\mathbf{1 9 4 7}$, deux ans après son retour à Paris. Entre le moment où elle avait quitté la petite ville de Bellins (Marne) et la date de notre rencontre, il faut bien admettre qu'elle mena, avec cette étrange Stellovski, une vie relativement heureuse.

(Frantext: François-Régis Bastide, Les adieux, Paris, Gallimard, 1956, p. 2II-2I2)

Le tour sans adjectif s'est construit à partir de la locution avec inversion. On est en effet passé de toujours est-il vrai/certain/sûr... que à toujours est-il que mais il est toujours vrai/certain/sûr... que n'a pas donné * il est toujours que. Dans cette nouvelle forme, des incises restent possibles comme l'atteste l'exemple [23] (je reviens sur le début de ce récit), ainsi que des variations temporelles que nous illustrons en [24], [25] et [26] :

[24] Ce coq-à-l'âne était charmant, et n'est pas trop clair ici; mais toujours est-il et sera-t-il que cette dame était une scrupuleuse personne.

(Frantext: Comte Honoré de Mirabeau, Lettres originales écrites du donjon de Vincennes pendant les années I777, I778, I779, I780 [1780], Paris - Strasbourg - Londres, Garnery Treuttel - De Boffe, I792, p. 33I)

[25] Toujours était-il que leur pauvre espoir, une fois encore, s'était envolé.

(Pierre Benoit, Bethsabée [1938]; cité par Le Bidois, 1952: 105)

[26] Toujours fut-il que Bernard l'accepta.

(Pierre Benoit, Axelle [1928]; cité par Le Bidois, 1952: 105)

Le degré de figement est cependant plus élevé avec toujours est-il que puisque nous ne pouvons plus supposer que l'adjectif soit sous-entendu d'un point de vue syntaxique dans la mesure où l'on trouve massivement sur Google la suite toujours est-il qu'il est vrai que ${ }^{18}$ - qu'on ne peut pas restituer sous la forme toujours est-il vrai qu'il est vrai que - ainsi que les formes toujours est-il qu'il est certain/évident/sûr... que.

Et surtout, apparaît un nouveau contexte. On s'aperçoit en effet que la forme sans adjectif toujours est-il que n'impose pas nécessairement une discordance modale entre ce qui suit la locution et le segment de texte qui la précède immédiatement. La

18. Et si l'on peut imaginer les suites (non attestées): (i) toujours est-il qu'il est faux que p/toujours est-il qu'il n'est pas vrai que p, on exclut: (ii) ??toujours est-il vrai qu'il est faux que p/??toujours est-il vrai qu'il n'est pas vrai que $p$. 
locution signifie alors «pour en revenir à ce que je disais» (Buchi, 2007) et confère tout simplement à ce qui précède le statut de digression, ce qui n'est pas forcément le cas lorsqu'il y a discordance modale. Soit l'exemple [27] où la digression figure en italique:

[27] - Veux-tu m'écouter. Je n'allais pas chaque fois au cours de dessin. Je parle d'un cours de sténo-dactylo. Papa m'avait laissé entendre qu'il me trouverait une place de secrétaire, et il fallait savoir la sténo. J'ai essayé, mais comme tu me déconseillais cette place, j'ai lâché le cours. J'y ai été trois fois - par miracle! J'y ai rencontré une jeune fille, une jeune femme, plutôt... enfin, elle a trois ans de plus que moi... qui vivait grâce à la gentillesse d'un type de cinquante ans. Le type la considérait un peu comme sa fille. Il était veuf et il avait perdu une fille qui lui ressemblait. Toujours est-il qu'elle m'a ouvert son cœur, et c'était triste. Je l'ai revue. Je séchais les cours... je préparais des dessins d'avance: cruches et pivoines... je n'aurais jamais osé t'en ouvrir la bouche avant qu'elle ne se soit décidée, d'elle-même, à quitter ce pauvre type, à faire place nette, à repartir à zéro. Elle m'adore, maman, et je l'adore, et tu l'adoreras, et elle est libre, et notre roulotte a l'esprit large, et mon rêve est de vous conduire chez elle, toi, papa, Léo, dès demain.

(Frantext: Jean Cocteau, Les parents terribles [1938], Paris, Gallimard, 1955, p. 206-207)

Nous reviendrons sur ce type d'emploi ci-dessous.

Maintenant que nous avons présenté succinctement la formation diachronique de la locution - formation qui exhibe son fonctionnement de dicto puisque toujours porte explicitement sur la vérité, la validité de $p$ dans les expressions il est toujours vrai/certain... que $p$, toujours est-il vrai/certain que $p$ - nous pouvons passer à l'examen des formes sous lesquelles se présentent le point de vue initial, le point de vue réaffirmé, et les séquences intercalées.

\section{Examen et classement des contextes typiques}

\subsection{Examen de la fin de la séquence intercalée}

Si on cherche à classer les cotextes gauches communs aux deux formes avec ou sans adjectif (soulignés dans les exemples ci-dessous), quatre structures syntaxiques sont identifiables (voir également Hansen, 2008: 200):

- Quand la locution suit l'expression d'une incertitude, celle-ci est exprimée:

I. sous la forme d'une subordonnée conditionnelle et/ou concessive :

[28] [...] et quand même beaucoup de nous en seraient privés jusqu'à un certain point, toujours est-il vrai que c'est dans cette puissance que consiste la raison, le bon sens [...].

(Frantext: Antoine-Louis-Claude Destutt de Tracy, Éléments d'idéologie. 3. Logique, Paris, Courcier, 1805, p. 324) 
[29] Qu'il y ait ou qu'il n'y ait pas là un motif sérieux de rejeter mon $\underline{\text { soupçon, toujours est-il que j’ai gardé un souvenir très vivant }}$ de cette peur.

(Frantext: Michel Leiris, La règle du jeu. 2. Fourbis [1955], Paris, Gallimard, 1997, p. 24)

2. par une modalisation atténuative (probabilité épistémique):

[30] Les mêmes personnes peuvent sans doute concourir à former différents corps. Mais toujours est-il vrai qu'une représentation extraordinaire ne ressemble point à la législature ordinaire. $\mathrm{Ce}$ sont des pouvoirs distincts.

(Frantext: Emmanuel-Joseph Sieyès, Qu'est-ce que le Tiers-État? [1789], Paris, Société de l'histoire de la Révolution française, I888, p. 72)

[3I] Pendant plus d'une semaine qu'elle a réfléchi, et elle m'a laissé bien tranquille... Elle avait dû en confier deux mots à sa mère de mes accès... Toujours est-il qu'elles insistaient moins pour me garder... (Frantext: Louis-Ferdinand Céline, Voyage au bout de la nuit [1932], Paris, Gallimard, 2000, p. 456)

3. au moyen d'une interrogative ou d'une série d'interrogatives directes:

[32] Beauvoir plut à la dame, il la trouva fort à son goût; peut-être s'aimèrent-ils? En prison l'amour va si vite! Commirent-ils quelque imprudence? Le sentiment qu'ils eurent l'un pour lautre dépassa-t-il les bornes de cette galanterie superficielle qui est presque un de nos devoirs envers les femmes? Beauvoir ne s'est jamais franchement expliqué sur ce point assez obscur de son histoire; mais toujours est-il constant que le commandant se crut en droit d'exercer des rigueurs extraordinaires sur son prisonnier. Beauvoir, mis au donjon, fut nourri de pain noir, abreuvé d'eau claire, et enchaîné suivant le perpétuel programme des divertissements prodigués aux captifs.

(Frantext: Honoré de Balzac, La muse du département [1843], Paris, Gallimard, 1976, p. 684)

[33] On chercha en vain le philosophe norvégien. Une colique l'avait-elle saisi? Avait-il eu peur de manquer le train? Un aéroplane était-il venu le chercher? Avait-il été emporté dans une Assomption? Toujours est-il qu'il avait disparu sans qu'on eût le temps de s'en apercevoir, comme un Dieu.

(Marcel Proust, Sodome et Gomorrhe, t. II, chap. II, in À la recherche du temps perdu, t. V, Paris, Gallimard, 1922; cité par Le Bidois, 1952: 105) 
- Enfin, lorsqu'il y a aveu d'ignorance, celui-ci est typiquement exprimé avec un verbe suivi d'une subordonnée interrogative indirecte ${ }^{19}$ :

[34] Nous ignorons si elle fut le motif d'une autre tentative du ministère: toujours est-il sûr qu'il essaya de persuader aux évêques de soumettre à sa censure, avant de les publier, leurs lettres pastorales et leurs mandements. (Frantext: Félicité de Lamennais, De la Religion considérée dans ses rapports avec l'ordre politique et civil: $2^{e}$ partie, Paris, Bureau du Mémorial catholique, I826, p. 20)

[35] Ils ont pris en grippe mon fils Charles, qui est doux comme un mouton; je ne sais pas ce qu'ils ont contre lui, toujours est-il qu'ils lui font souffrir le martyre.

(Frantext: Champfleury, Les souffrances $d u$ professeur Delteil [1853], Paris, M. Lévy, 1857, p. 13)

Dans tous ces cas de figure répertoriés, la construction inversée, avec ou sans adjectif, signale une discordance modale dans le sens où elle introduit ce dont le locuteur est sûr en opposition à ce dont il est moins sûr, ou pas sûr du tout. À chaque fois, elle indique la forte prise en charge ${ }^{20}$ du locuteur relativement à ce qui suit. Pour mesurer le degré de prise en charge (ou d'engagement) du locuteur, nous utilisons le test de l'implication (Lenepveu et Gosselin, 2013; Lenepveu, 20I4). Une locution adverbiale comme à première vue marquera une prise en charge minimale (à première vue $p$ n'implique pas $p$ ) tandis que toujours est-il que, de toute évidence ou encore je sais que signalent une forte prise en charge: toujours est-il que $p$ implique $p$; de toute évidence $p$ implique $p$; je sais que $p$ implique $p$. En revanche, ce qui précède immédiatement la locution dans ces exemples n'est pris en charge que très faiblement, d'où un effet de sens éventuellement concessif ${ }^{21}$.

Mais toujours est-il que autorise également, comme on l'a vu au paragraphe précédent (exemple [27]), un nouveau contexte où l'on n'observe plus cette discordance modale. Toujours est-il que est alors marqueur de «fin de digression» dans la

19. Les différents procédés peuvent se combiner; par exemple, une interrogative directe peut être suivie d'un aveu d'ignorance: Nous a-t-elle trabis depuis?'Je ne sais; toujours est-il qu'un soir, rentrant dans ma maison, japerçus un homme dans la rue qui semblait observer ma fenêtre avec curiosité (Frantext: Champfleury, Les Bourgeois de Molinchart [1855], Paris, M. Lévy, I859, p. 317).

20. Notons que si la prise en charge maximale est généralement associée à un haut degré de croyance du locuteur relativement au contenu propositionnel, toujours est-il que interagit avec d'autres marques de jugement (verbes et adverbes modaux). Ainsi, toujours est-il que signale la forte prise en charge par le locuteur de la probabilité de la venue de Paul dans l'exemple Toujours est-il que Pierre viendra peut-être demain. Par ailleurs, un marqueur de haut degré de croyance comme il est certain que $p$ n'implique pas une forte prise en charge (il est certain que $p$ n'implique pas $p$ ). Sur la notion de prise en charge, et le débat qui s'y rapporte, voir en particulier Coltier et al. (2009).

21. On comprend que toujours est-il que puisse dans ce type de contexte se paraphraser par en tout cas, une locution dont la caractéristique est de signaler une position de repli sur ce qui est sûr, assuré (Haillet, 2007: 57-58). 
mesure où c'est sa présence qui impose d'interpréter ce qui précède immédiatement comme une digression ${ }^{22}$; la locution signale le retour à l'essentiel, c'est-à-dire à ce qui est pertinent pour la suite de la narration. Cette digression peut d'ailleurs faire l'objet d'un commentaire métadiscursif de la part du narrateur comme dans l'exemple [36] (faut pas que je vous ennuie avec ces détails):

[36] - [...] Ils se sont engueulés, si ça peut vous rendre service, monsieur le commissaire. - Engueulés?

- Oui, enfin, j'ai l'impression. Diaz et lui, enfin, ils ont échangé comme qui dirait des insultes. Dans la chambre de Diaz, c'est-à-dire. Mais je les ai entendus, c'est-à-dire que jeetais monté à l'étage parce que le sanitaire était obstrué, faut pas que je vous ennuie avec ces détails mais toujours est-il que j'étais avec mon jonc et je les entendais qui s'injuriaient à travers la porte.

(Frantext: Jean-Patrick Manchette, Nada [1972], Paris, Gallimard, 1999, p. 14I)

Ainsi se dégagent, au plan de la structuration textuelle, deux cas de figure qui correspondent respectivement aux deux emplois, «concessif» et "thématique», dégagés par Hansen (2004) et Buchi (2007):

- Quand il y a discordance modale, la locution a, en aval, une portée qu'on pourrait qualifier de cadrative ${ }^{23}$ (Charolles, 1997; Sarda et Charolles [éd.], 2012), elle fournit en effet un nouveau critère d'interprétation pour ce qui suit, à savoir que le jugement qui va être réaffirmé est fortement pris en charge par le locuteur.

- En l'absence de discordance modale, la locution joue un rôle de cadratif «rétroactif» dans le sens où elle impose de lire ce qui précède comme une digression, et signale la fin de cette digression.

Mais dans les deux configurations, nous considérons à l'instar de Blumenthal (1980: 126-127) et à sa suite, avec Nøjgaard (1992: 485), que toujours est-il que signale la plus grande pertinence ${ }^{24}$ de ce qui va suivre par rapport à ce qui précède

22. La digression est prise ici comme une figure de discours qui consiste en un changement temporaire de sujet (de topique discursif). Nous retenons la définition de Fouquelin (I990 [1555]) qui souligne, comme le remarque Milhe Poutingon (2012: 15), la présence de formules d'ouverture et de fermeture : «Digression est une interruption de propos et changement en un autre, non étrange du tout: laquelle est quelquefois un peu plus longue, quelquefois plus courte. La Digression plus longue est volontiers prémunie d'une petite et brève préface, de peur que inconsidérément et sans y penser nous semblions aller du coq à l'âne: et à la fin d'icelle on a de coutume mettre une petite clausule signifiant la retraite et rentrée au propos, duquel on était sorti». Il semble qu'un marquage en aval au moyen de toujours est-il que soit suffisant pour interpréter le segment de texte précédant cette locution comme une courte digression.

23. Toujours est-il que en tant que cadratif s'apparenterait ici aux cadratifs à portée véridictionnelle (Charolles, 1997).

24. Son rôle dans l'agencement global du discours a été étudié par Blumenthal (1980) dans le cadre de la "functional sentence perspective» (FSP), toujours est-il que ayant pour propriété de renverser la hiérarchie des valeurs communicatives en faisant passer au premier plan ce qui était thématique. L'analyse est reprise par Nøjgaard (I992: 485) pour qui «l'effet de sens [...] est de nous dire que seule la dernière partie de 
immédiatement (séquence intercalée) quant à la véracité des propos dans le premier cas, et en tant qu'objet (topique) de discours dans le second cas de figure ${ }^{25}$.

Il faut alors prendre en compte la relation entre le point de vue initial et le point de vue réaffirmé pour comprendre le rôle de marqueur de cohésion de toujours est-il que.

\subsection{Examen de la relation entre point de vue initial et point de vue réaffirmé}

Ici encore, différents cas de figure sont à considérer.

Le point de vue réaffirmé reprend une information antérieure en la paraphrasant, ce que nous illustrons avec le dialogue ci-dessous où le point de vue réaffirmé est une reformulation du point de vue initial ${ }^{26}$ :

[37] - Voyez-vous, on est convaincu qu'un sénateur a reçu mission de se rendre ici, et que le gouvernement va vous destituer. Cela m'est revenu de plusieurs côtés.

- Je l'ai entendu dire aussi, observai-je.

- Qui a parlé de cela? demanda la gouvernante toute rouge.

- Vous voulez dire: qui en a parlé le premier? Je n'en sais rien. Toujours est-il qu'on en parle, et même beaucoup. Le public ne s'est pas entretenu d'autre chose dans la journée d'hier. Tout le monde est très sérieux, quoiqu'on n'ait encore aucune donnée positive. Sans doute les personnes plus intelligentes, les gens plus compétents se taisent, mais parmi ceux-ci plusieurs ne laissent pas d'écouter.

(Bibliothèque électronique du Québec: Fiodor Dostoïevski, Les possédés, trad. V. Derély, Paris, E. Plon, I886, t. II, $3^{\mathrm{e}}$ partie, chap. II)

Le point de vue réaffirmé reprend une information antérieure en la développant, en l'expliquant ou en la justifiant, ce dont on peut rendre compte au moyen des relations rhétoriques de Mann et Thompson (1988). Ainsi le point de vue réaffirmé dans l'exemple [38] va-t-il entretenir une relation de justification avec le point de vue initial («c'est parce qu'il entendit du bruit qu'il lui parut évident que...»):

[38] Lorsque Aliocha, dans le vestibule, se fit annoncer par la femme de chambre qui lui avait ouvert, il lui parut évident qu'on connaissait déjà au salon son arrivée

l'ensemble reste entièrement pertinente». La locution est rapprochée «d'adverbiaux qui ne résument ce qui précède que pour en noter le manque de pertinence pour l'enchaînement argumentatif», telle la locution quoi qu'il en soit qui annonce «une information plus pertinente, donc plus importante» ou encore la locution anaphorique en tout cas (Nøjgaard, 1992: 486-488).

25. En signalant la moindre pertinence de ce qui précède, toujours est-il que aurait alors un fonctionnement comparable à des expressions, identiques de par la position de l'adverbe et du sujet pronominal, du type encore faut-il admettre que, encore faut-il préciser que, encore ne s'agit-il que de, etc. qui remettent en cause la pertinence d'un dire antérieur (Fuchs, 1997: 299-309).

26. On note que l'assertion Je l'ai entendu dire aussi est elle-même une reformulation de Cela m'est revenu de plusieurs côtés. 
(peut-être l'avait-on aperçu de la fenêtre) ${ }^{27}$; toujours est-il qu'il entendit du bruit, des pas précipités résonnèrent avec un frou-frou de robes, deux ou trois femmes avaient dû s'échapper. Aliocha trouva étrange que son arrivée produisît une telle agitation. On le fit entrer aussitôt au salon, une grande pièce meublée avec élégance, qui n'avait rien de provincial [...].

(Bibliothèque électronique du Québec: Fiodor Dostoïevsky, Les frères Karamazov, trad. H. Mongault, Paris, Gallimard, 1994, ${ }^{\text {re }}$ partie, chap. IX)

Le point de vue réaffirmé peut être également une explicitation d'une information inférable à partir du point de vue initial ou bien encore présupposée par ce point de vue. Voir les exemples [39] et [40]:

[39] - [...] Mais où sont les antennes dont vous parliez?

- Les antennes! dit Legrand, qui s’échauffait inexplicablement; vous devez voir les antennes, j'en suis sûr. Je les ai faites aussi distinctes qu'elles le sont dans l'original, et je présume que cela est bien suffisant.

- À la bonne heure, dis-je; mettons que vous les ayez faites; toujours est-il vrai que je ne les vois pas.

(Bibliothèque électronique du Québec: Edgar Allan Poe, Histoires extraordinaires, trad. C. Baudelaire, Paris, M. Lévy, 1856, «Le scarabée d'or»)

[40] Patricia Martin: «Depuis quand les fleurs ont-elles un langage?», la question est de Paul.

Alain Baraton : Connaissez-vous Charlotte de La Tour? Personne ne connait Charlotte de La Tour. Cette femme écrit un ouvrage en i819. Cet ouvrage commence par cette phrase: «Heureuse la jeune fille qui ignore les joies du monde et ne connait pas de plus douce occupation que l'étude des plantes». Vous vous dites: «Aucune femme n'aurait la bêtise d'écrire ça». Eh bien, vous avez raison. Car derrière Charlotte de La Tour se cache Louis-Aimé Martin. C'est un homme qui, sous un pseudonyme féminin, a écrit le premier langage des fleurs. Il s'avère que ce livre est un succès. Moralité, en I844, deuxième édition de Charlotte de La Tour, mais qui n'est plus écrit par un dénommé Martin, mais par Louise Cortambert, qui s'est pas embêtée. Elle a repris le bouquin et elle a gagné une fortune avec. Et toujours est-il que, depuis, les fleurs ont un langage. Et il est vrai que offrir des roses, offrir des mimosas [...].

(France Inter, le I4 février 2015 , à 07 h 52 )

Dans l'exemple [39], le point de vue réaffirmé se donne comme la reprise d'une information inférable à partir d'une question («du fait que le narrateur demande où sont les antennes, on peut inférer qu'il ne les voit pas»). Tandis qu'il est la reprise, dans l'exemple [40], d'une information présupposée tout d'abord par la question

27. La parenthèse peut être interprétée ici comme une digression, marquée en amont comme en aval par des bornes ponctuantes ouvrantes et fermantes. Sur les relations analogiques entre digression et parenthèse, nous renvoyons de nouveau aux travaux de Milhe Poutingon (2012: 15). 
Depuis quand les fleurs ont-elles un langage?, et ensuite par l'assertion C'est un bomme qui, sous un pseudonyme féminin, a écrit le premier langage des fleurs, à savoir que les fleurs ont un langage.

Enfin, il existe un emploi spécifique à la narration, dans lequel la locution introduit un événement nouveau qui s'inscrit à la suite d'un événement antérieur. Ainsi, dans l'exemple [4I], une idée va-t-elle tout d'abord «naître» (C'est ainsi qu'un beau soir naquit l'idée de partir ensemble) pour ensuite "prendre corps» (Toujours est-il qu'elle prit corps):

[4I] C'est ainsi qu'un beau soir naquit chez eux l'idée de partir tous ensemble pour New York en excursion de vacances, et d'aller voir par eux-mêmes s'il n'y avait rien de neuf à tirer de ce qu'on savait déjà.

Qui formula le premier cette idée? C'est un point resté obscur et qui servit longtemps de thème aux discussions du docteur et de $\mathrm{M}$. Bredejord; chacun prétendait la priorité. Sans doute ils l'eurent en même temps, car, à force de la cultiver, Erik devait en avoir saturé l'air ambiant. Toujours est-il qu'elle prit corps, qu'elle fut définitivement adoptée, et qu'au mois de septembre de l'année suivante, les trois amis, accompagnés d'Erik, s'embarquèrent à Christiania pour les États-Unis.

(Arvensa Éditions: Jules Verne et André Laurie, L'épave du Cynthia, Paris, J. Hetzel, I885, chap. VIII)

D'où, pour résumer, cinq types de relations entre le point de vue initial et le point de vue réaffirmé:

I. (quasi-)paraphrase (exemple [37]);

2. développement, explication, justification (exemple [38]);

3. explicitation d'une information inférable (exemple [39]);

4. explicitation d'une information présupposée (exemple [40]);

5. enchaînement narratif (exemple [4I]).

\section{Conclusion}

On a montré comment le fonctionnement pragmatique de la locution toujours est-il que prenait son origine dans sa structure syntactico-sémantique, et comment la valeur de dicto de toujours est-il que pouvait être rattachée à la valeur de persistance de toujours, hypothèse qui se confirme si on décrit l'évolution de la formation de la locution, à partir de Frantext. Lorsqu'il y a discordance modale, la persistance marquée par toujours porte sur le maintien du point de vue (du jugement), tandis qu'elle porte uniquement sur le maintien du topique discursif (de l'objet du discours) en l'absence de discordance modale.

L'examen systématique des contextes de gauche très larges de la locution (qui peuvent s'étendre sur plusieurs pages), aussi bien en synchronie qu'en diachronie (pour les formes non encore figées du type il est toujours vrai/certain... que $p$ et 
toujours est-il vrai/certain... que p), nous permet d'affirmer que dans tous les cas (et pas seulement dans les emplois dits "thématiques», qui sont récents et restent relativement marginaux), il y a retour à un topique antérieur, à la suite d'une séquence intercalée. La valeur de persistance de toujours sert précisément à indiquer le maintien de ce topique (et, dans les cas de discordance modale, du point de vue initial) en dépit des doutes et/ou des digressions qui ont introduit une interruption dans la continuité topicale et/ou modale, sous la forme d'une séquence intercalée.

Toutefois, ce retour à un topique initial n'est pas toujours explicite ni immédiatement repérable. Sa mise au jour a nécessité une étude spécifique des relations sémantiques et/ou rhétoriques entre les deux segments connectés par-delà la séquence intercalée. Nous avons identifié des relations I) de (quasi-)paraphrase, 2) de développement, explication, justification, 3 ) d'explicitation d'une information inférable, 4) d'explicitation d'une information présupposée et 5) d'enchaînement narratif.

Toujours est-il que s'apparente ainsi aux locutions adverbiales susceptibles de marquer l'aspect de dicto dans le sens où elles signalent l'évolution dans le temps d'un jugement (à y regarder de plus près, tout compte fait, tout bien considéré, etc.) (Lenepveu, 2OIO, 20II, 20I4). Mais à la différence de celles-ci qui ont pour caractéristique de signaler un changement de point de vue, toujours est-il que exprime le maintien d'un jugement initial, à l'instar des locutions le fait est que ou reste que (tout au moins dans certains de leurs emplois).

\section{Références}

Blumenthal, P. 1980. La syntaxe du message. Application au français moderne. Tübingen: M. Niemeyer.

BUCHI, É. 2007. Sur la trace de la pragmaticalisation de l’adverbe toujours («Voyons toujours l'apport de la linguistique historique»). Langue française I54: IIO-I25.

Cadiot, A., Ducrot, O. et Nguyen, T.-B. 1985. Sous un mot, une controverse : les emplois pragmatiques de toujours. Modèles linguistiques 7 (2): I05-I24.

Charolles, M. 1997. L'encadrement du discours - univers, champs, domaines et espaces. Cabiers de recherche linguistique 6: $\mathrm{I}^{-} 73$.

Charolles, M. 2003. De la topicalité des adverbiaux détachés en tête de phrase. Travaux de linguistique 47: $\mathrm{II}-49$.

Coltier, D., Dendale, P., De Brabanter, P. 2009. La notion de prise en charge: mise en perspective. Langue française $162:$ 3-27.

Craige, R. 2oio. Topics. In K. von Heusinger, C. Maienborn et P. Portner (éd.), Semantics: An International Handbook of Natural Language Meaning. Berlin - Boston: Mouton de Gruyter. Vol. 2: 1908-I934.

Damourette, J. et Pichon, E. 1987. Des mots à la pensée. Essai de grammaire de la langue française. Paris: Éditions d'Artrey. Vol. 7: I9II-I940.

Fouquelin, A. 1990 [1555]. La rhétorique française. In F. Goyet (éd.), Traités de poétique et de rbétorique de la Renaissance. Paris : Librairie générale française. 
FRANCKeL, J.-J. 1989. Étude de quelques marqueurs aspectuels du français. Genève - Paris: Droz - Champion.

Fuchs, C. 1988. Encore, déjà, toujours: de l'aspect à la modalité. In N. Tersis et A. KinM (éd.), Temps et aspects (Actes du colloque CNRS, Paris, 24-25 octobre 1985). Louvain - Paris : Peeters - SELAF : $135^{-1}$ I48.

Fuchs, C. 1997. Petite promenade au pays des paraphrases: à propos des énoncés en encore faut-il... In D. Miéville et A. BerRendonner (éd.), Logique, discours et pensée: mélanges offerts à Jean-Blaise Grize. Berne - Berlin - New York - Paris: P. Lang: 279-3II.

GuimIER, C. 1996. Les adverbes du français: le cas des adverbes en -ment. Gap - Paris: Ophrys.

Guimier, C. I997. La place du sujet clitique dans les énoncés avec adverbe initial. In C. Fuchs (éd.), La place du sujet en français contemporain. Louvain-la-Neuve : Duculot: 43-96.

Haillet, P.-P. 2007. Pour une linguistique des représentations discursives. Bruxelles : De BoeckDuculot.

Hansen, M.-B. M. 2004. La polysémie de l'adverbe toujours. Travaux de linguistique 49: $39-55$.

Hansen, M.-B. M. 2008. Particles at the Semantics/Pragmatics Interface: Synchronic and Diachronic Issues. A Study with Special Reference to the French Phasal Adverbs. Amsterdam - New York - Paris : Elsevier.

Jacobs, J. 200I. The Dimensions of Topic-Comment. Linguistics 39 (4): 64I-68I.

LE BidoIs, R. 1952. L'inversion du sujet dans la prose contemporaine (1900-1950). Paris: Éditions d'Artrey.

Lenepveu, V. 20io. De l'expression du point de vue à l'anticipation textuelle: le rôle de à première vue. Discours 7: I-24. En ligne à l'adresse suivante: http://discours.revues. org/8II5.

Lenepveu, V. 2OII. À première vue, marqueur d'aspect de dicto. Journal of French Language Studies 2I (3): 38I-400.

Lenepveu, V. 20I4. Aspect de dicto et structuration informationnelle dans les textes. Lingvistice Investigationes 37 (I): I29-155.

Lenepveu, V. et Gosselin, L. 20I3. Les marqueurs d'aspect de dicto: à première vue, à y regarder de plus près, au bout du compte, tout compte fait... In C. NorÉN et al. (éd.), Modalité, évidentialité et autres friandises langagières. Berne - Berlin - Bruxelles: P. Lang: 227-269.

Mann, W. C. et Thompson, S. A. 1988. Rhetorical Structure Theory: Toward a Functional Theory of Text Organization. Text 8 (3): 243-28I.

Marandin, J.-M. 1988. À propos de la notion de topique de discours. Langue française 78 : $67-88$.

MARTIN, R. 1987. Langage et croyance: les univers de croyance dans la théorie sémantique. Bruxelles: P. Mardaga.

Milhe Poutingon, G. 2oi2. Poétique du digressif. La digression dans la littérature de la Renaissance. Paris: Classiques Garnier.

Morel, M.-A. 1996. La concession en français. Paris - Gap: Ophrys. 
Muller, C. 1999. Encore et toujours les modifieurs aspectuels: de encore à toujours. In M. PléNAT et al. (éd.), L'emprise du sens: structures linguistiques et interprétations. Mélanges de syntaxe et de sémantique offerts à Andrée Borillo par un groupe d'amis, de collègues et de disciples. Amsterdam: Rodopi : 217-237.

Nemo, F. 2000. Enfin, encore, toujours entre indexicalité et emplois. In A. Englebert et al. (éd.), Actes du XXII congrès international de Linguistique et de Philologie romanes (Bruxelles, 23-29 juillet 1998). Tübingen: Niemeyer. Vol. 7: 499-5II.

Nguyen, T.-B. i986a. Toujours est-il. Revue romane 2I (2): 192-207.

Nguyen, T.-B. 1986b. À propos des emplois pragmatiques de toujours. Modèles linguistiques 8 : I23-I39.

NøjgaARD, M. 1992. Les adverbes français. Essai de description fonctionnelle. Copenhague: Munksgaard. Vol. I: Méthode et inventaire, connecteurs et adverbiaux connectifs.

NøLKe, H. 1994. Linguistique modulaire: de la forme au sens. Louvain - Paris: Peeters - Société pour l'information grammaticale.

SARDA, S. et Charolles, M. (éd.) 20I2. Travaux de linguistique: Les adverbiaux prépositionnels position, fonction et portée 64. Bruxelles: Duculot.

Traugott, E. C. et Dasher, R. B. 2002. Regularity in Semantic Change. Cambridge: Cambridge University Press.

Van Dijk, T. A. 1976. Sentence Topic and Discourse Topic. Papers in Slavic Pbilology I : 49-6I.

Van Kuppevelt, J. I995. Discourse Structure, Topicality and Questioning. Journal of linguistics 3I (I): 109-I47. 